\title{
Experimental Investigation of an Influence of Coupled Compressive Loading on Porcine Spine Specimens
}

\author{
Sandra MIKUCKYTË*, Vytautas OSTAŠEVIČIUS** \\ *Kaunas University of Technology, Studentu str. 56, 51424, Kaunas, Lithuania, E-mail: sandra.mikuckyte@ktu.lt \\ **Kaunas University of Technology, Studentu str. 56, 51424, Kaunas, Lithuania, E-mail: vytautas.ostasevicius@ktu.lt \\ crossref http://dx.doi.org/10.5755/j02.mech.26903
}

\section{Introduction}

The human spine is a structure which carries the weight of upper body, helps to maintain upright posture, allows moving, amortizes loadings, and protects the spinal cord. Any significant changes in this structure could lead to discomfort, loss of function or pain. The human spine shortens approximately by $1 \%$ of its height during the daily activities due to the influence of static and cyclic loads and returns to its primary height during the night rest due to rehydration of the intervertebral discs. In general, compression is considered a healthy loading condition for the disc as biologic remodelling may outbalance damage of the disc structure [1]. Also, changes of intervertebral disc height and intradiscal pressure govern by fluid fluctuations are necessary in order to ensure diffusion and convection of nutrients and metabolites. On the other hand, dynamic compression leads to increase in gene expression for collagens and aggrecan in the disc while static compression suppress it [2], but higher loading rate leads to greater stress decay and higher probability of disc herniation [3] and the study of an in vivo porcine model exposed to vibrational loading shows that dynamic axial stiffness of the intervertebral disc is frequency dependent [4], thus loading frequency contributes to spinal health as well as loading magnitude as both static and cyclic loads should not exceed the limits that would cause declined load distribution and worsened shock absorption due to reduced fluid content or residual structural changes.

While no statistically significant difference between ultimate axial compression strength of functional spinal units previously exposed to cyclic compression and controls were reported [5], repetitive movement of daily activities and cumulative low back loads are the risk factors of low back pain [6] and have damaging influence on the intervertebral discs [7]. Compression, compression together with flexion and/or extension and other loading scenarios are carried out in order to investigate the influence of repetitive movement on the intervertebral discs and vertebral bodies. For example, while no catastrophic failures were observed in flexed and to vibrational loading exposed specimens, internal damage such as delamination and disruption to the inner and mid-annular layer, tearing between the inner disc and endplate that could lead to initiation of further degenerative changes were detected [8].

Studies of combined axial compression and flexion/extension cyclic loading (up to 20000 cycles) with various compression magnitudes $(260-1500 \mathrm{~N})$ at physiological frequency $(0.33-1 \mathrm{~Hz})$ reveal that mechanical loadings may also be associated with an intervertebral disc herniation, degeneration and other issues. This type of loading which rep- resents common daily movement leads to decreased intradiscal pressure and partial disc herniation but no correlation between damage type and intradiscal pressure was yet determined [9]. Herniation is more likely to occur due to high cyclic flexion/extension moments than excessive compression force [10] and the higher degree of flexion leads to the statistically significant decrease of cycles the functional spinal units last to failure when holding of $9 \mathrm{~kg}$ load is simulated [11]. Also, survivability of the disc is higher when constant amplitude cyclic loading is applied compared with variable loading pattern [12]. Combined cyclic loading also increases lumbar spinal column laxity that contributes to instability and related spinal problems by increasing the natural zone length and decreasing slope in both adolescent cervine and elderly human cadaveric specimens [13]. Furthermore, it leads to decreased MRI (magnetic resonance imaging) signal in the growth zone of the superior vertebra, the inferior vertebrae and endplates and increased signal in the superior vertebral body that could be interpreted as a sign of fatigue as well as changes of chondrocytes structure in the endplates and growth zones and deformation of extracellular matrix [14]. High magnitude of cyclic flexion is more likely to cause vertebral fractures while more cycles of low magnitude load is more likely to lead to the injury of intervertebral disc [15].

Most of the studies use functional spinal units to analyse the influence of cyclic loading and just a few in silico studies that investigate the influence of cyclic loading on the entire lumbar spine, for example a study that presents transient analysis of the lumbar spine under compressive preload and sinusoidal axial load with the frequencies in the range of $3-15 \mathrm{~Hz}$ [16].

The goal of this study is to investigate the influence of cyclic compression and flexion, as these are the most common moves during the day, on the structural integrity and geometrical parameters such as height and cross-sectional area of the disc of the spinal specimens consisting of more than one intervertebral disc.

\section{Methods}

Two specimens consisting of four adjacent vertebrae and three intervertebral discs were separated from fresh healthy porcine spines which were obtained from a local abattoir. They were placed in plastic bags and fresh frozen at $-20^{\circ} \mathrm{C}$ in order to reduce an effect of dehydration and environment on their properties. Before testing, they were thawed in $0.9 \%(0.15 \mathrm{~mol} / \mathrm{l})$ saline overnight in a refrigerator $\left(+4^{\circ} \mathrm{C}\right)$ to allow rehydration. 


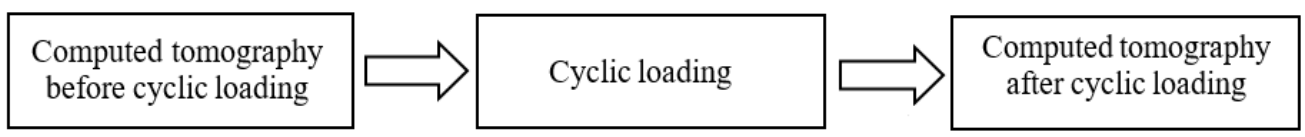

Fig. 1 Stages of the experimental study

This study was carried out in three principal stages: initial computed tomography (CT) scanning of the specimens, cyclic loading and the second CT scanning of the specimens to in order to study the influence of cyclic loading on the specimens (Fig. 1). Between the scans and cyclic loading, the specimens were kept in moist cloths in order to prevent dehydration.

Computed tomography is a medical diagnostic tool that allows visualising the inside structures of the human body and is performed by generating cross-sectional images (slices) of the scanned body. A fan-shaped beam of X-ray produced by a rotating X-ray source is attenuated by the scanned object which it travels through, and this attenuation is captured by a rotating X-ray detector (Fig. 2) and converted into a digital signal for computed reconstruction. Measurements taken during one rotation result in a single $2 \mathrm{D}$ image. As the table where the scanned object is placed moves, a sequence of these images is produced, and this sequence can be used to create cross-sectional or three-dimensional views of the entire scanned object. LightSpeed VCT (GE Healthcare) computed tomography system was used in this study. The CT dose index $\mathrm{CTDI}_{\mathrm{vol}}$ was $14.74 \mathrm{mGy}$ and the dose-length product DLP was $899.74 \mathrm{mGy} / \mathrm{cm}$. A slice thickness of $0.625 \mathrm{~mm}$ was chosen for this study.

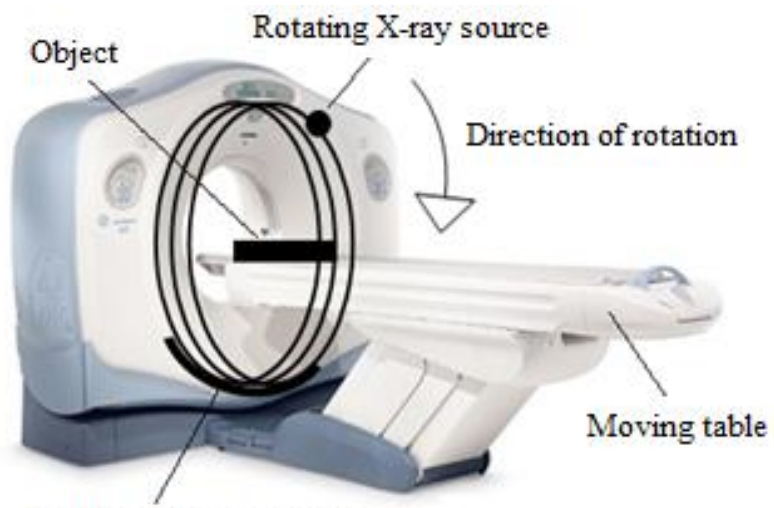

Rotating X-ray detector

Fig. 2 Scheme of computed tomography imaging (picture of LightSpeed VCT computed tomography system used to create this scheme was taken from [17])

Following the first CT scanning, upper part of cranial vertebra and lower part of cranial vertebra of the specimens were placed in specially designed holders and inserted into a linear-torsion static and fatigue testing machine Instron ElectroPuls E10000T. Experimental setup for cyclic loading of the specimens are given in Fig. 3. All testing was carried out in room temperature. The specimens were loaded with 6,000 cycles of combined compression and flexion/extension load with the peak compression of $500 \mathrm{~N}$ and 1000 N. All the characteristics of the loads applied to the specific specimen are given in Table 1.

After cyclic loading, CT scanning was repeated in order to capture the current condition of the specimens. During the primary and repeated scans, the specimens were placed in the same position in order to ensure easier comparison of the images.

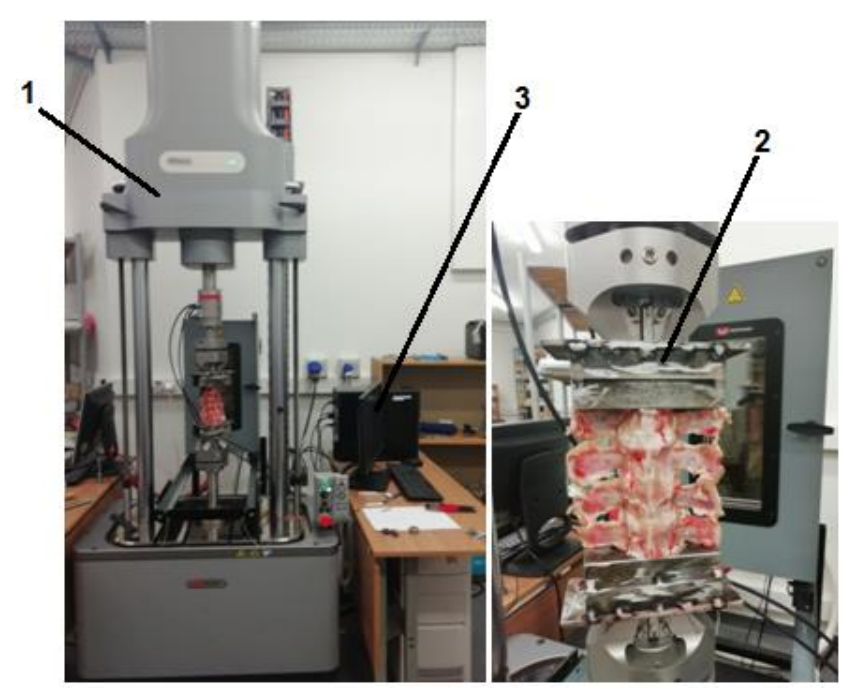

Fig. 3 Experimental setup: 1 - Linear-Torsion static and fatigue testing machine Instron ElectroPuls E10000T; 2 - specimen holder; 3 - PC

Table 1

Loading data of the specimens

\begin{tabular}{|l|l|l|}
\hline Specimen No. & 1 & 2 \\
\hline Max cycle load, N & 500 & 1000 \\
\hline Min cycle load, N & 100 & 100 \\
\hline Loading frequency, Hz & 2 & 5 \\
\hline
\end{tabular}

All the CT images of the horizontal sections of the specimens were saved as DICOM (Digital Imaging and Communications in Medicine) files. As the sagittal plane of spinal segments was not parallel to the vertical plane during the scanning, first of all, the MATLAB (Mathworks Inc.) code was used to rotate all the obtained CT images by $32^{\circ}$, while other information contained in these files was preserved. Then, CT images were processed with Sante DICOM Viewer 3D Pro (SanteSoft LTD) software, and the sagittal sections of the specimens were created and ready for further analysis (Fig. 4).
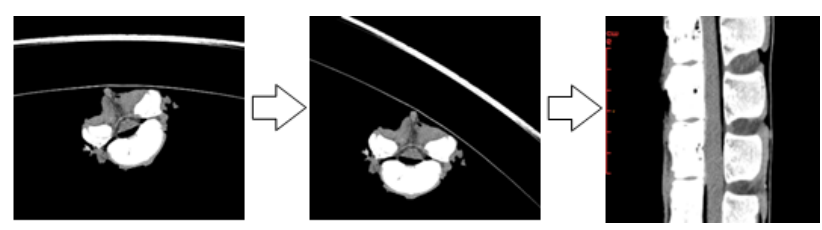

Fig. 4 Scheme of DICOM images processing: original image (left), rotated image (middle), midsagittal section of the specimen (right)

The height and the section area of the discs before and after loading were measured in the midsagittal section view. The height of the discs was measured at five locations of each intervertebral disc (Fig. 5) approximately equally spaced between the left and the right edges of the midsagittal section of the disc. One-way analysis of variance (ANOVA) with a significance level $\alpha=0.05$ was used for the statistical analysis of geometrical changes of the discs. 


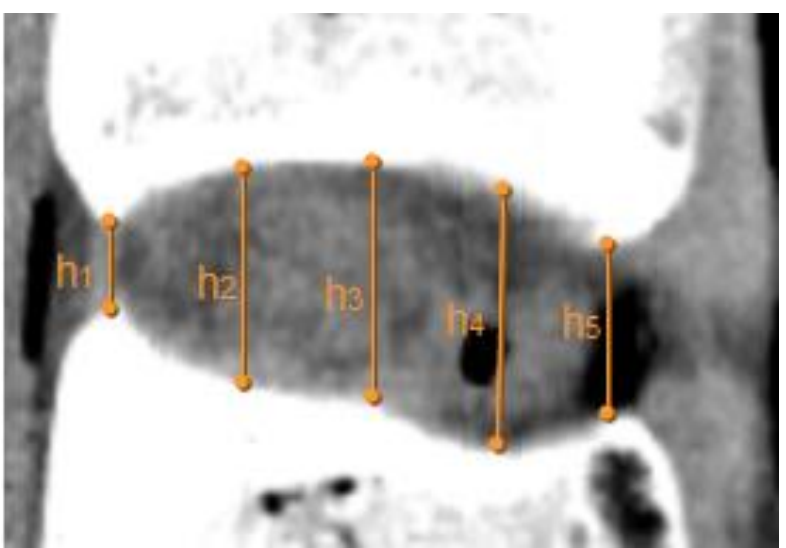

Fig. 5 Locations of measurement of intervertebral disc height

\section{Results}

Apparent deformations of intervertebral discs were observed during the loading cycle when, under maximum compression, the height of the discs decreased, and the discs themselves became more convex (Fig. 6).

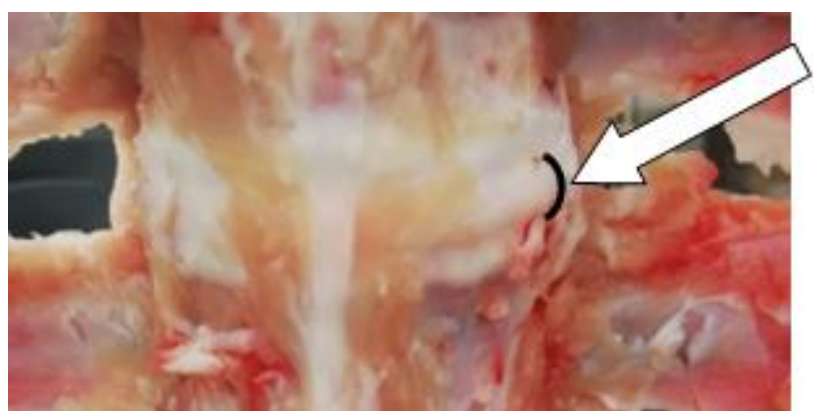

Fig. 6 Deformation of intervertebral disc during cyclic loading

A significant decrease of the specimen height (Fig. 7) was observed during loading, mainly due to the loss of the fluid content within intervertebral discs. For the first 500 cycles the decrease of the height of both specimens were almost the same. Later, $2 \mathrm{~Hz}$ and $500 \mathrm{~N}$ loading led to the higher decrease of the height of the specimen than $5 \mathrm{~Hz}$ and $1000 \mathrm{~N}$ loading. In total, the height of the first specimen decreased by $9.535 \mathrm{~mm}$, and the height of the second specimen decreased by $7.958 \mathrm{~mm}$. During the interval between cycles 500 and 6000 , the height of the first specimen decreased by $5.779 \mathrm{~mm}$ and the height of the second specimen decreased by $4.208 \mathrm{~mm}$. In total, the difference of the height of the two specimens immediately after the loading was $1.577 \mathrm{~mm}$.

The midsagittal section view of the first specimen is given in Fig. 8, and the midsagittal section view of the second specimen is given in Fig. 9. No obvious damage to the specimens was observed when comparing images before and after the loading. The anterior and posterior lines of vertebral columns remained smooth and intact. Also, no displacement of any vertebra over any other vertebra that is common due the degenerative disc disease is seen. The condition of the vertebrae was observed by changing the software setting to the bone level window, and no fractures of the bones were observed.

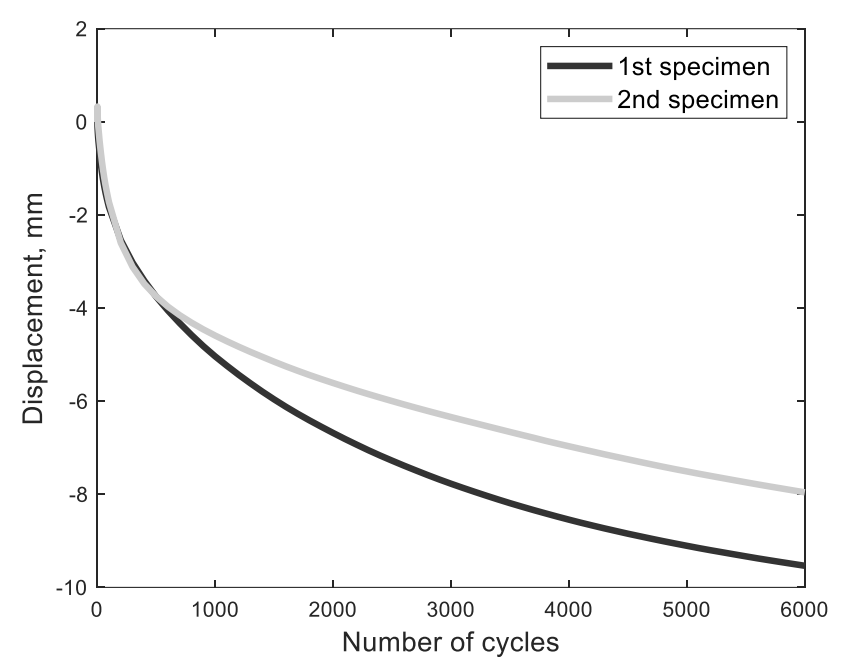

Fig. 7 Decrease of specimens' height

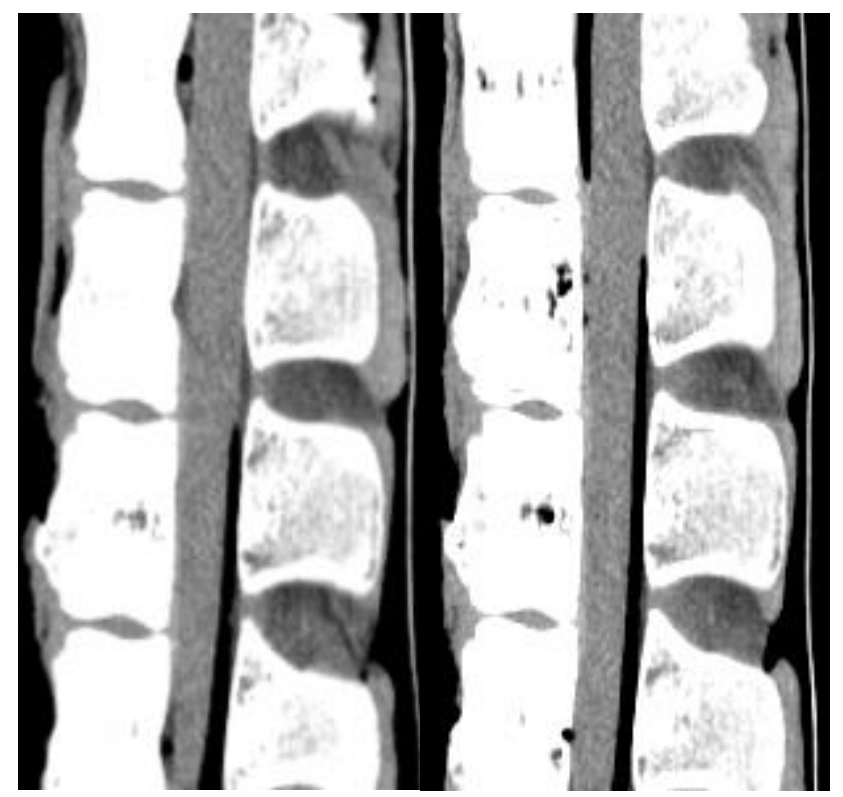

Fig. 7 Comparison of 1st specimen CT scans: before loading (left) and after loading (right)

The results of the averaged values of the measurements of the heights and midsagittal cross-section areas of the intervertebral discs are summarised in Table 2. There is a statistically significant difference between the height of the intervertebral discs before and after loading in both the first specimen $(p=0.0224)$ and the second specimen $(p=$ $0.0155)$ with the lowest disc of both specimens decreasing the most and obviously losing the highest water content. The positive change of the middle disc of the fist specimen was found as, at three out of five measuring locations of this disc, increases of the height after loading were found. It may be explained by residual angular displacement of the vertebrae connected by this intervertebral disc.

Changes of the cross-sectional area also demonstrate the same tendency: the cross-sectional area of the lowest disc decreased the most. The increase of the cross-sectional area of the middle intervertebral disc of the first specimen may most likely be explained by the same reason as the increase of the height of this disc.

The results of the measurement of the changes of intervertebral discs after cyclic loading show that, even in short specimens of three sequenced intervertebral discs, the lowest discs are affected the most. 
Table 2

Changes of disc height and section area ("-" represents the reduction of disc height $\Delta h$ or cross-sectional area $\Delta A$ )

\begin{tabular}{|c|c|c|c|c|}
\hline Disc & $\Delta h, 1^{\text {st }}$ spec., $\mathrm{mm}$ & $\Delta h, 2^{\text {nd }}$ spec., $\mathrm{mm}$ & $\Delta A, 1^{\text {st }}$ spec., $\mathrm{mm}^{2}$ & $\Delta A, 2^{\text {nd }}$ spec., mm ${ }^{2}$ \\
\hline Upper & -0.508 & -0.119 & 0 & -0.100 \\
\hline Middle & 0.242 & -0.545 & 0.260 & -0.050 \\
\hline Lower & -2.230 & -2.005 & -0.120 & -0.400 \\
\hline
\end{tabular}

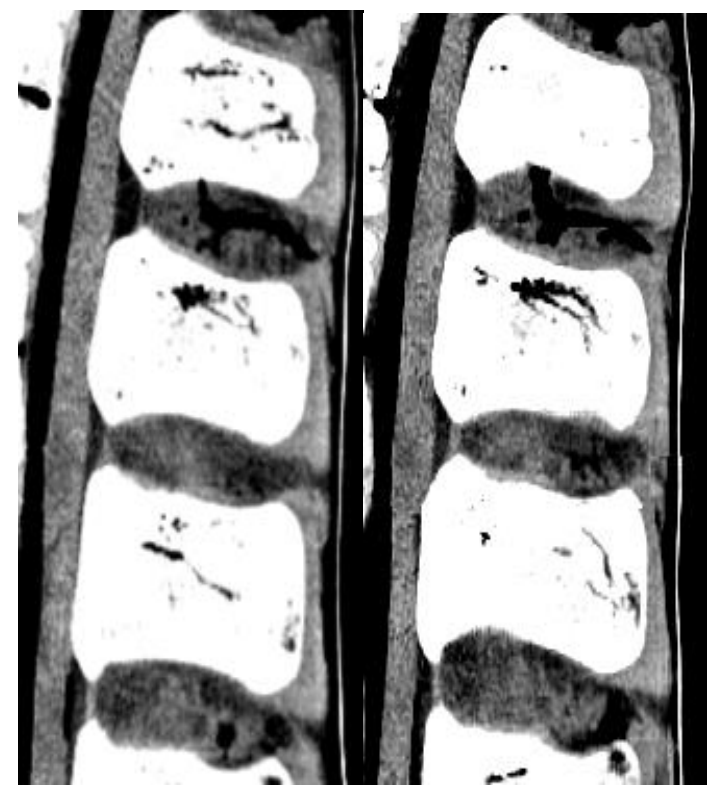

Fig. 8 Comparison of 2nd specimen CT scans: before loading (left) and after loading (right)

\section{Discussion}

In this in vitro study porcine specimens consisting of 3 intervertebral disc were exposed to cyclic compression and flexion an this allowed not only to investigate the loading influence on the intervertebral discs but also to compare how the changes of the intervertebral disc depends on its position in the specimen.

The result that loads of $500 \mathrm{~N}$ and $1000 \mathrm{~N}$ did not cause disintegration of the structure of the specimens or any other obvious damage complies with reporting of other studies, for example, Thoreson et al. [5], where cyclic compression with maximal magnitude of $1000 \mathrm{~N}$ was not reported to cause failure of functional spinal unit consisting of single intervertebral disc. The specimens demonstrated increasing deformation during the loading. $8 \mathrm{~Hz}$ loading frequency led to lower decrease of the height of the specimen even though compression of $1000 \mathrm{~N}$ was applied to this specimen in contrary to compression of $500 \mathrm{~N}$ applied to the other specimen. However, a thorough evaluation of optimal cyclic loading frequencies was not a goal of this study so the further investigation to understand this phenomenon is necessary.

It is known that the lowest discs of the human lumbar spine are the ones that carry most of the load and, that is why, they are the biggest one. This study also confirms this statement as the lowest intervertebral discs in both quite short specimens if compared with the entire lumbar spine were deformed the most as their height and midsagittal section area decreased the most.

Some limitations of this study must be also noticed. As this was considered a primary study for choosing suitable loading magnitudes and frequencies in order not to break the specimens, only two specimens were used in the study.
Small number of specimens does not allow to draw statistically reliable quantitative conclusions, but it still allows to observe the tendencies. Due to very limited availability of fresh frozen cadaveric spines, porcine specimens were chosen for this study. Animal models are widely used as an alternative as they can be chosen by the desired age group and health condition in order to ensure more uniform material properties. The porcine model was chosen for this study as it said to be the most resembling the human spine.

\section{Conclusions}

Coupled cyclic loading of $500 \mathrm{~N}$ or $1000 \mathrm{~N}$ compression and flexion did not induce intervertebral disc herniation or other apparent damage to spine specimens. Maximal height loss was observed in the lowest intervertebral discs of the specimens. This once again confirms that lower part of the spine such as intervertebral discs L4-L5 and L5$\mathrm{S} 1$ are the least prone to the injuries and degeneration due to disturbed nutrition and loss of water content.

\section{References}

1. Korecki, C. L.; MacLean, J. J.; Iatridis, J. C. 2008. Dynamic compression effects on intervertebral disc mechanics and biology, Spine 33(13): 1403-1409. https://doi.org/10.1097/BRS.0b013e318175cae7.

2. Wang, D. L.; Jiang, S. D.; Dai, L. Y. 2007. Biologic response of the intervertebral disc to static and dynamic compression in vitro. Spine 32(23): 2521-2528. https://doi.org/ 10.1097/BRS.0b013e318158cb61.

3. Tsai, K. H.; Lin, R. M.; Chang, G. L. 1998. Rate-related fatigue injury of vertebral disc under axial cyclic loading in a porcine body-disc-body unit, Clinical Biomechanics 13(1): S32-S39. https://doi.org/10.1016/S0268-0033(98)80134-4.

4. Kaigle, A.; Ekstrom, L.; Holm, S.; Rostedt, M.; Hansson, T. 1998. In vivo dynamic stiffness of the porcine lumbar spine exposed to cyclic loading: influence of load and degeneration, Journal of Spinal Disorders 11(1): 65-70.

5. Thoreson, O.; Ekstrom, L.; Holm, S.; Hellstrom, M.; Sward, L. 2010. The immediate effect of repeated loading on the compressive strength of young porcine lumbar spine, Knee Surgery, Sports Traumatology, Arthroscopy 18: 694-701. https://doi.org/10.1007/s00167-009-1001-z.

6. Coenen, P.; Kingma, I.; Boot, C. R.; Twisk, J. W. R.; Bongers, P. M.; van Dieen, J. H. 2013. Cumulative low back load at work as a risk factor of low back pain: a prospective cohort study, Journal of occupational rehabilitation 23: 11-18.

https://doi.org/ 10.1007/s10926-012-9375-z.

7. Desmoulin, G. T.; Pradhan, V.; Milner, T. E. 2020. Mechanical aspects of intervertebral disc injury and implications on biomechanics, Spine 45(8): E457-E464. 
https://doi.org/ 10.1097/BRS.0000000000003291.

8. Wade, K. R.; Schollum, M. L.; Robertson, P. A.; Thambyah, A.; Broom, N. D. 2016. Vibration really does disrupt the disc, Spine 41(15): 1185-1198. https://doi.org/ 10.1097/BRS.0000000000001594.

9. Noguchi, M.; Gooyers, C. E.; Karakolis, T.; Noguchi, K.; Callaghan, J. P. 2016. Is Intervertebral disc pressure linked to herniation?: an in-vitro study using a porcine model, Journal of Biomechanics 49(9):1824-1830. https://doi.org/ 10.1016/j.jbiomech.2016.04.018.

10. Callaghan, J. P.; McGill, S. M. 2005. Intervertebral disc herniation: studies on a porcine model exposed to highly repetitive flexion/extension motion with compressive force, Clinical biomechanics (Bristol, Avon) 16(1): 28-37. https://doi.org/ 10.1016/s0268-0033(00)00063-2.

11. Gallagher, S.; Marras, W. S.; Litsky, A. S.; Burr, D. 2005. Torso flexion loads and the fatigue failure of human lumbosacral motion segments, Spine 30(20): 22652273. https://doi.org/ 10.1097/01.brs.0000182086.33984.b3.

12. Zehr, J. D.; Tennant, L. M.; Callaghan, J. P. 2019. Examining endplate fatigue failure during cyclic compression loading with variable and consistent peak magnitudes using a force weighting adjustment approach: an in vitro study, Ergonomics 62(10): 1339-1348. https://doi.org/ 10.1080/00140139.2019.1648879.

13. Gale, N. C.; Zeigler, S. L.; Towler, C.; Mondal, S.; Issen, K. A.; Mesfin, A.; Michalek, A. J.; Kuxhaus, L. 2018. Increased lumbar spinal column laxity due to lowangle, low-load cyclic flexion may predispose to acute injury, JOR Spine 1(4): 1-8. https://doi.org/10.1002/jsp2.1038.

14. Thoreson, O.; Ekstrom, L.; Hansson, H. A.; Todd, C.; Witwit, W.; Sward Aminoff, A.; Jonasson, P.; Baranto, A. 2017. The effect of repetitive flexion and extension fatigue loading on the young porcine lumbar spine, a feasibility study of MRI and histological analyses, Journal of Experimental Orthopaedics 4, 16. https://doi.org/10.1186/s40634-017-0091-7.

15. Parkinson, R. J.; Callaghan, J. P. 2009. The role of dynamic flexion in spine injury is altered by increasing dynamic load magnitude, Clinical Biomechanics 24(2): 148-154. https://doi.org/10.1016/j.clinbiomech.2008.11.007.

16. Fan, W.; Guo, L.-X. 2017. Influence of different frequencies of axial cyclic loading on time-domain vibration response of the lumbar spine: a finite element study, Computers in Biology and Medicine 86: 75-81. https://doi.org/10.1016/j.compbiomed.2017.05.004.

17. Bctechnical. VTC-64 [online]. [viewed: 21 November 2018]. Available from:

https://bctechnical.com/systems/vct-64/.
S. Mikuckytė, V. Ostaševičius

EXPERIMENTAL INVESTIGATION OF AN
INFLUENCE OF COUPLED COMPRESSIVE
LOADING ON PORCINE SPINE SPECIMENS

S u m m a r y

The human spine shortens approximately by $1 \%$ of its height during the daily activities and returns to its primary height during the night rest. Cyclic loading is important in order to ensure diffusion and convention of the nutrients and metabolites within the intervertebral discs. On the other hand, cyclic loading could lead to the damage of the intervertebral discs and the vertebra bodies if the magnitude and frequency of the loads applied to the spine exceed the allowable limits. As most of the in vitro studies that investigate the influence of cyclic loading deal with functional spinal units consisting of single intervertebral disc the purpose of this study is to investigate an influence of cyclic compression and flexion on the structural integrity and geometrical parameters of the spinal specimens consisting of more than one intervertebral disc.

Two specimens consisting of four adjacent vertebrae and three intervertebral discs were scanned by using computed tomography then loaded with combined cyclic compression and flexion and then scanned for the second time in order to capture the current condition of the specimens. Obtained images were used to evaluate the changes of structural integrity and geometrical parameters of the discs.

A significant decrease of the specimen height was observed during loading, mainly due to the loss of the fluid content within intervertebral discs. In total, the difference of the height of the two specimens immediately after the loading was $1.577 \mathrm{~mm}$. No obvious damage to the specimens was observed when comparing images before and after the loading. A statistically significant differences between the height of the intervertebral discs before and after loading in both the first specimen ( $p=0.0224)$ and the second specimen ( $p=0.0155)$ were calculated with the lowest disc of both specimens decreasing the most and obviously losing the highest water content. The cross-sectional area of the lowest disc in both specimens also decreased the most. This once again confirms that lower part of the spine such as intervertebral discs L4-L5 and L5-S1 are the least prone to the injuries and degeneration due to disturbed nutrition and loss of water content.

Keywords: intervertebral disc, cyclic loading, computed tomography, porcine spine.

Received June 29, 2020

Accepted January 17, 2021 